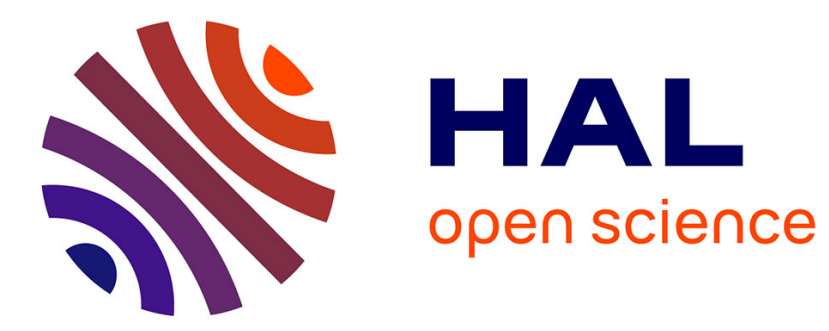

\title{
Le don gratuit. Le cas d'un établissement public
}

Yvan Barel, Sandrine Frémeaux

\section{To cite this version:}

Yvan Barel, Sandrine Frémeaux. Le don gratuit. Le cas d'un établissement public. Gérer et Comprendre. Annales des Mines, 2008, (94), pp.80-89. hal-00765468

\section{HAL Id: hal-00765468 \\ https://hal.science/hal-00765468}

Submitted on 9 Sep 2013

HAL is a multi-disciplinary open access archive for the deposit and dissemination of scientific research documents, whether they are published or not. The documents may come from teaching and research institutions in France or abroad, or from public or private research centers.
L'archive ouverte pluridisciplinaire HAL, est destinée au dépôt et à la diffusion de documents scientifiques de niveau recherche, publiés ou non, émanant des établissements d'enseignement et de recherche français ou étrangers, des laboratoires publics ou privés. 

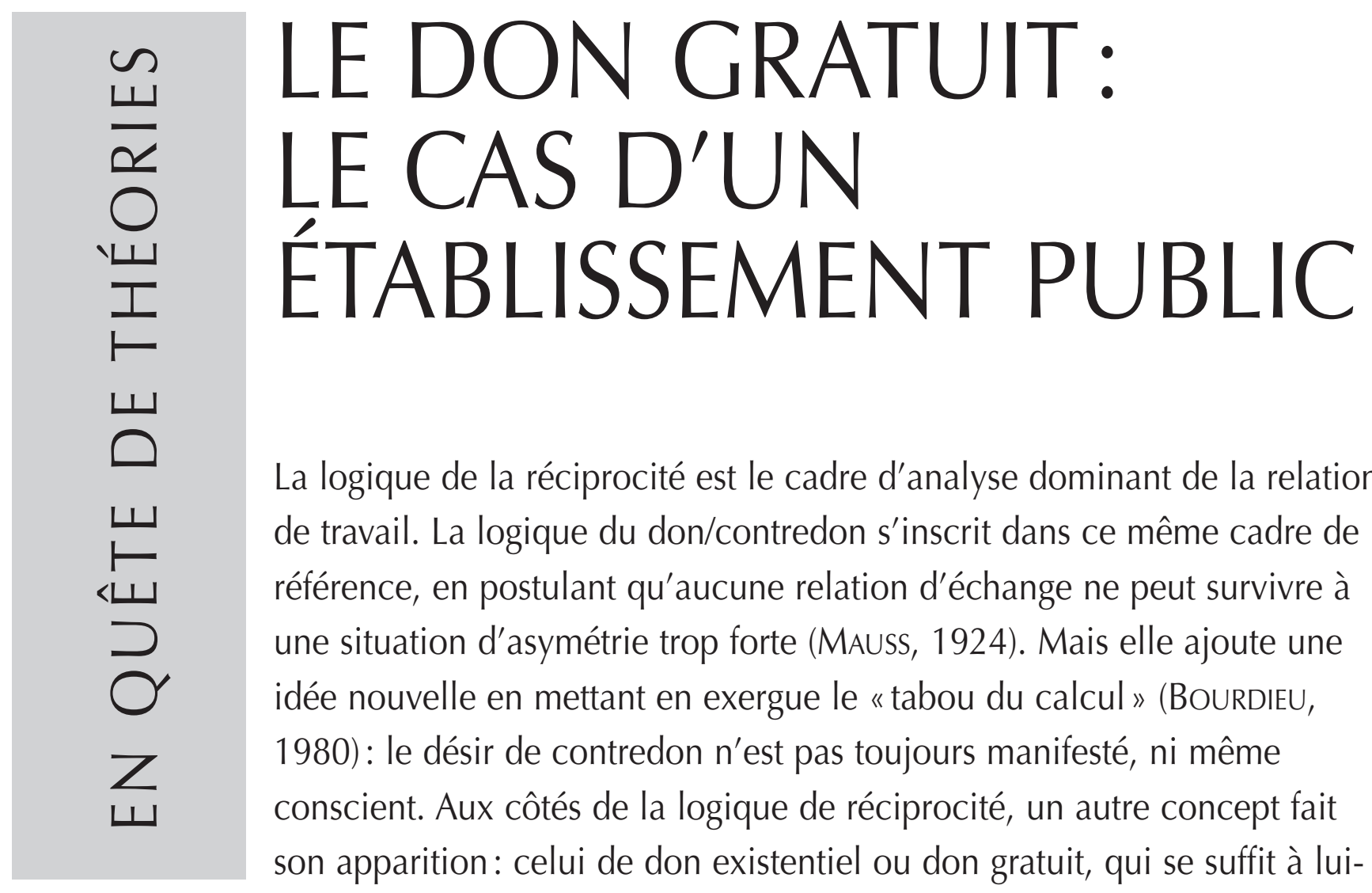

La logique de la réciprocité est le cadre d'analyse dominant de la relation de travail. La logique du don/contredon s'inscrit dans ce même cadre de référence, en postulant qu'aucune relation d'échange ne peut survivre à une situation d'asymétrie trop forte (MAuss, 1924). Mais elle ajoute une idée nouvelle en mettant en exergue le «tabou du calcul» (BOURDIEU, 1980): le désir de contredon n'est pas toujours manifesté, ni même conscient. Aux côtés de la logique de réciprocité, un autre concept fait son apparition: celui de don existentiel ou don gratuit, qui se suffit à luimême et dont les travaux de Callzé et Godbout (1992), puis de Dumond (2007) ont révélé l'importance. Déconnecté d'une quelconque attente de retour, le don existentiel est-il ce qui donne sens au travail?

Des expériences de don ont été exprimées par des salariés d'un CROUS (Centre Régional des OEuvres Universitaires et Scolaires), organisme public français ayant pour vocation de faciliter la vie des étudiants dans de nombreux domaines (restauration, logement, bourses, action sociale et culturelle, ouverture sur l'international). L'étude a été menée à l'occasion de la construction d'un projet d'établissement. Si, lors des entretiens semi-directifs réalisés auprès d'un échantillon transversal de trente-sept salariés, certains récits témoignent d'un attachement à la logique de réciprocité marchande, d'autres récits, au contraire, sont des témoignages de don aux étudiants. Cette analyse montre l'importance, pour un grand nombre de salariés, du rapport personnel, libre, non stratégique aux usagers, dans les entreprises de service public.

Par Yvan BAREL, Maître de conférences HDR en Sciences de gestion, LEMNA (Laboratoire d'Économie et de Management de Nantes-Atlantique), et Sandrine FREMEAUX, Professeur associé, Audencia Nantes École de management.

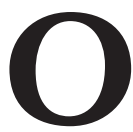
n a beaucoup reproché à l'organisation taylorienne de mépriser le sens du travail. À l'opposé des anciens modèles hiérarchiques de direction, l'entreprise contemporaine s'affiche comme un lieu de coopération, de responsabilité et de pouvoir décentralisé. S’appuyant sur les travaux de DURKHEIM, Weber, Parsons et Aron, Courpasson (2000) remet en question cette image, qu'il juge trompeuse, et montre que le discours actuel de gestion libérale des ressources humaines cache un dispositif de mise en 
conformité de l'individu. Chaque salarié est amené, selon cet auteur, à "se rendre visible, aimable, en un mot attractif, non pas en cultivant sa compétence distinctive, mais en faisant connâ̂tre sa conformité aux demandes locales des managers». Le sentiment d'avoir à intérioriser des contraintes liées au travail subsiste, voire s'est accru, depuis une vingtaine d'années (FourNiER, BOURASSA et BÉJI, 2003). La question du sens prêté au travail par les salariés est donc loin d'être anodine.

La réciprocité, que la théorie de l'échange (GOULDNER, 1960; BLAU, 1964) place au cœur de la relation d'emploi, est-elle, à elle seule, de nature à donner sens au travail? La réciprocité postule qu'aucune relation d'échange ne peut survivre longtemps à une situation d'asymétrie trop forte. Non que l'échange se doive d'être symétrique, mais l'asymétrie, en tant qu'elle ne peut pas être maintenue dans le temps à l'identique, est le moteur de la poursuite de la relation. Elle éveille une recherche de réciprocité, qui est précisément «l'attitude symétrique d'autrui».

Marcel MAUSS, dont les travaux sur le fonctionnement des sociétés archaïques ont été publiés sous le titre «Essai sur le don» dans la revue L'Année sociologique, en 1923-1924, a affiné l'analyse de la logique de réciprocité en élaborant le concept d' "échange-don» également appelé "don réciproque», qui met en évidence trois dimensions de l'échange: les obligations de donner, de recevoir et de rendre. L'originalité des travaux de Mauss est de ne pas vouloir séparer les biens et les rites qui sont l'objet de l'échange du sens et des symboles qui leur sont attachés. En utilisant le concept de "phénomène social total», Mauss propose de voir l'échange comme un «tout».

Ce concept de "phénomène social total» a été également mobilisé pour appréhender la relation de travail. Lentreprise donne, afin de faire naître chez les salariés le sentiment de devoir rendre ce qu'ils ont reçu, voire d'aller au-delà de la mission dévolue. C'est l'avènement de la logique du don/contredon (MAUSS, 1967; Derrida, 1992; CAIllé, 1994; Durkheim, 1996; CORDONNIER, 1997), laquelle est encore une théorie de l'échange. Certes, elle ajoute aux théories classiques de l'échange une idée nouvelle, que BOURDIEU appelle le "tabou du calcul» (1980). Le désir de contredon est tabou. Mais la logique du don/contredon n'exclut pas la norme de réciprocité; au contraire, elle l'intègre, puisqu'un salarié qui accomplit un don peut attendre, en retour, une attention bienveillante de la part de l'entreprise (BLAU, 1964; EISENBERGER et alii, 2001).

La présente étude vise à mieux comprendre ce qui fait la spécificité du don, c'est-à-dire cette absence de droit (TESTARD, 2001), mais aussi de désir (Eustache, 2001) d'obtenir une contrepartie. Le don qui se suffit à lui-même existe-t-il ? La relation de travail est-elle l'occasion pour les salariés de vivre des expériences de don qui soient déconnectées d'une quelconque attente de retour? Se peut-il que le don, rendu possible par une organisation souple et respectueuse des préoccupations de chacun, motive les salariés et donne sens à leur travail ?

\section{LES LIMITES DE LA LOGIQUE DE RÉCIPROCITÉ}

La logique de réciprocité est le cadre d'analyse dominant de la relation d'emploi, parce que la plupart des chercheurs en sciences de gestion se refusent à considérer que le salarié puisse agir sans aspirer, en échange, à quelque compensation. Les théories de l'implication, comme les théories de la motivation (SCHEIN, 1965; ROUSSEAU, 1990), partent du principe que le salarié envisage son travail comme un moyen d'atteindre des résultats (ThéveneT et VACHETTE, 1992). Les théories classiques de l'implication voient la relation entre individu et organisation comme une série d'échanges et d'attentes réciproques (GOULDNER, 1960; Homans, 1958). Les travaux de BECKER (1960) ont enrichi cette approche échangiste ou transactionnelle, mettant en exergue ce que l'individu pourrait perdre en quittant l'organisation, c'est-à-dire le coût d'opportunité de la participation. Il en va de même des théories de la motivation, qui mettent en évidence le résultat recherché par l'individu. Avec les théories substantialistes de la motivation, le salarié veut satisfaire des besoins, que MASLOW (1954) a hiérarchisés, en partant des besoins les plus primaires (besoins physiologiques) pour aller jusqu'aux besoins les plus élevés (besoins d'épanouissement et de réalisation). Les théories du processus de la motivation mettent en évidence le processus cognitif de perception des résultats possibles de l'action (valence), des conséquences prévisibles des résultats (instrumentalité) et du lien perçu entre l'effort et la performance (expectance). D'autres approches ont complété ces théories: la théorie de l'équité, qui voit dans l'iniquité ce qui motive la personne à tout faire pour la réduire (ADAMS, 1965); ou encore, la théorie du besoin de réussite, qui part de l'idée que le principal déterminant de la motivation, c'est le désir de réussite (MC Clelland et alii, 1953). Ces théories ont pour point commun de faire du travail un instrument permettant d'obtenir un résultat. FRYER et PAYNE (1984) avaient déjà remarqué que le travail est déterminé par un but qui se définit au-delà du plaisir engendré par son exécution. BRIEF et NORD (1990) ajoutent que, dans toutes ses acceptions, le travail est perçu comme une activité qui a un but ("purposeful activity»). Ces théories de l'obtention conduisent à faire de l'instrumentalité une référence centrale et croissante de l'action organisée.

Or, l'action instrumentale ne se suffit pas à ellemême. Elle "ne peut, en elle-même, trouver le sens qui pourrait la légitimer. Certes, accomplir une action dans un but donné n'apparâ̂t pas absurde et peut même parfois regorger de sens. À condition, toutefois, que le but 
se suffise à lui-même. Sinon, ce but n'est, à nouveau, qu'un moyen, dont le sens est à rechercher dans le but ultime quil sert. [...] Mais, lorsque l'existence est emportée par la certitude d'une maîtrise impossible et par la conscience quil faut s'en remettre au destin, estil encore possible de concevoir une action instrumentale? La gratuité devient alors nécessaire." (DUMOND, 2005). prise ce qui permet aux salariés de trouver un sens ou une "part d'identité", opposent l'affirmation de soi (qui se caractérise par le pouvoir) et le dépassement de soi (qui signifie la bienveillance). En mettant en exergue le conflit qui oppose entre eux ces deux domaines motivationnels, Schwartz et Sagig semblent s'interroger sur un lien possible entre l'affirmation de soi et le dépassement de soi et ils excluent de leur ana-

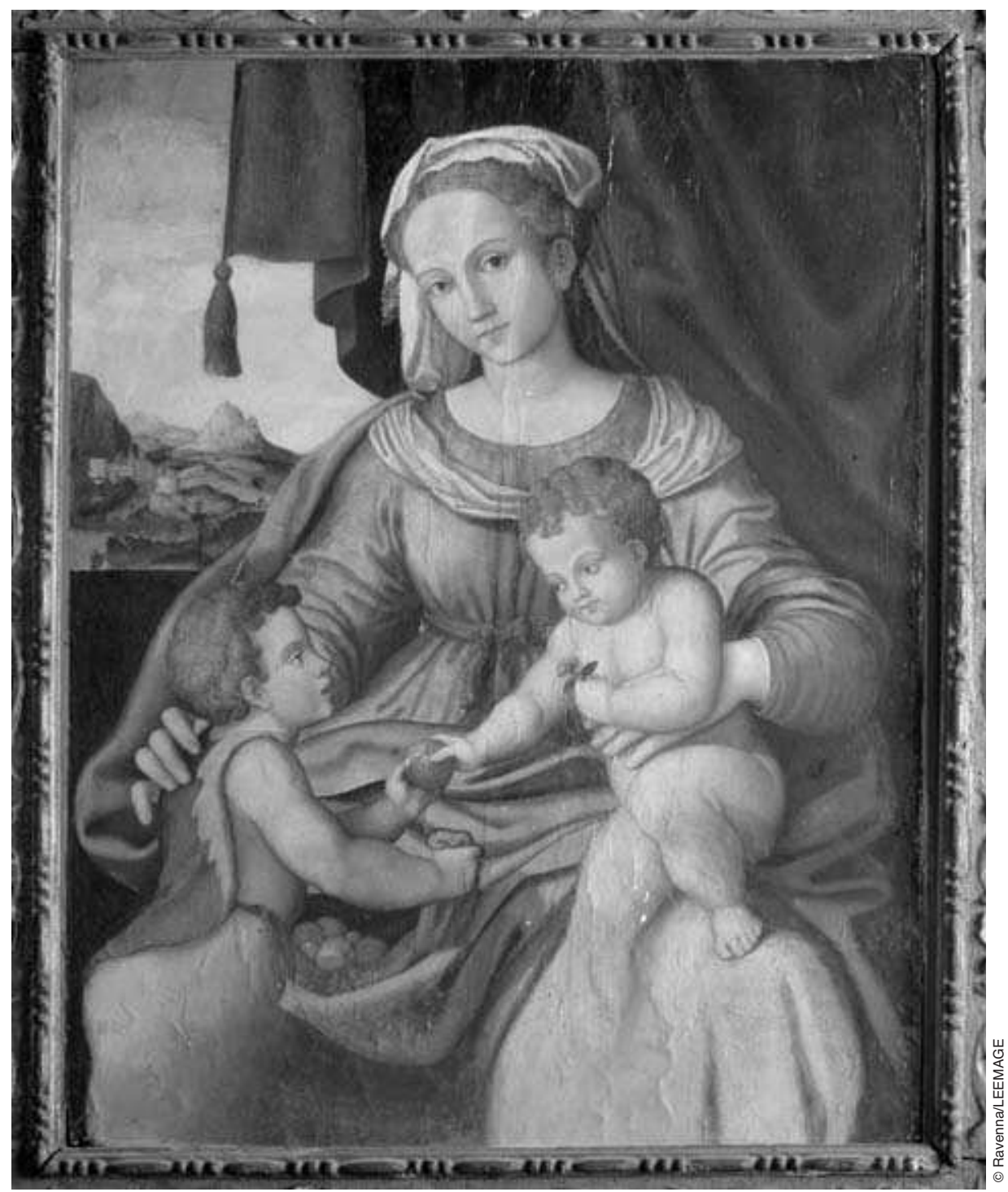

«Ce qui fait la gratuité des actes de don, ce n'est pas leur absence d'intérêt, mais le fait que leur finalité se limite à leur réalisation ", Madone dite La Charité. (École italienne, XV siècle Bologne, église San Stefano).

Certaines théories récentes ont tenté de s’éloigner de la logique de l'obtention. Dans la théorie des ancres de carrières, développée par SCHEIN (1975), qui met en perspective les talents, les motivations et les valeurs pour expliquer les processus à l'origine des orientations professionnelles, on voit apparaitre une ancre de carrière qui serait le service ou le dévouement. Mais aucun lien n'est établi entre le service et le don. Dans la théorie de l'universalité de la structure des valeurs, SCHWARTZ et SAGIG (1995), qui voient dans l'entre- lyse le don gratuit, qui relève d'un tout autre registre. Par l'étude du don gratuit, la présente étude tente non seulement de dépasser une analyse de l'action instrumentale, mais aussi d'y intégrer la recherche du sens dans la relation de travail. Dès lors, elle exclut une approche désenchantée du don, qui a marqué les théories classiques sur l'altruisme et en vertu de laquelle le don sert l'intérêt mutuel d'un groupe dont les membres se sentent dans l'obligation d'opérer des sacrifices au profit de la communauté (BOURDIEU, 1994). Elle exclut 
également une approche, relationnelle, qui fait du don le moteur du lien social et, plus précisément, de la relation de travail, de la confiance (TRÉPO et DUMOND, 1998) ou de la coopération (DuMOND, 1997).

Le don gratuit ne peut être appréhendé qu'à travers l'acception existentielle du don, développée par GODBOUT (1992) et, plus récemment, par DuMOND (2000). Dans L'Esprit du don (1992), CAILlÉ et GODBOUT montrent que le don est porteur de sens parce qu'il témoigne, tout à la fois, du refus du rapport instrumental à autrui, de la joie de donner et de la portée affective des échanges sociaux. Penser le don comme ce qui donne sens à la relation de travail, c'est "rompre aussi bien avec les explications de la pratique humaine que propose l'utilitarisme qu'avec les diverses variantes du nietzschéisme; avec celles qui représentent le sujet humain comme un égoïste naturel, aussi bien quivec celles qui veulent voir en lui, au moins dans sa variante occidentale moderne, un assoiffé de pouvoir." (CAillé et Godbout, 1992, p. 27).

Ce qui signifie, en premier lieu, que le don "n'attend pas de retour et ignore le contredon" (BOLTANSKI, 1990). Le contredon n'est ni attendu, ni pris en compte. Ce qui différencie la logique du don de la logique marchande, c'est l'absence de droit, de garantie et d'espoir d'un bénéfice d'une quelconque nature. Le don est déconnecté du calcul et de l'intention, se traduisant par une sorte d' "abandon au flux du monde" (GODBOUT, 2000 , p. 12). Il est vrai que le retour peut exister (Dumond, 2005). Ainsi, le don n'exclut pas, par exemple, un bénéfice narcissique qui lui soit postérieur. De façon générale, il ne signifie pas une absence d'intérêt ou de plaisir. Mais «si l'on ne peut exclure que l'individu altruiste trouve une réelle satisfaction personnelle dans ses conduites de générosité, de bienveillance, de dévouement - et tel est, en effet, sans doute, le cas -, pareille satisfaction ou gratification n'est que le résultat indirect d'actions qui ne sétaient pas donné cet objectif pour fin première." (TERESTCHENKO, 2005, p. 11).

Ce qui signifie, en second lieu, que le don est libre. Un don qui serait obligatoire, contraignant ou même attendu ne serait plus un don. Parce que le don est libre et laisse l'autre parfaitement libre de rendre ou de ne pas rendre, "il va tout autant à l'encontre du principe public d'égalité que du principe marchand de l'équivalence.»(GODBOUT, 1992, p. 84).

Ainsi, le don n'est assurément pas une intention, un calcul, un sacrifice, une morale, un idéal ou une action particulière, dont on ne comprendrait pas les raisons. Un critère, tiré de la littérature psychologique, peut d'ores et déjà être mis en avant: la "présence à soi», que ROGERS (1998) définit comme la capacité d'être pleinement ce que l'on est, et non ce que les autres attendent ou exigent de nous. Cette "présence à soi» avait été évoquée par la philosophe Simone WeIL (1955) énonçant que le travail ne peut être porteur de sens que s'il est "conscient» et permet au salarié d'être. On retrouve cette même notion dans les analyses de Fromm (1975): l'homme "doit acquérir le sentiment d'être capable de faire quelque chose, de remuer quelquiun, de marquer son empreinte ou, pour utiliser le mot anglais le plus juste d'être effective (efficace). On se sert aujourd'hui de ce mot à propos d'un orateur ou d'un vendeur" efficace "pour désigner celui qui réussit à obtenir des résultats. Mais c'est une altération de sens originel de to effect (effectuer) du latin exfacere, faire [...]. C'est, en dernière analyse, la preuve quion est. Le principe peut être exprimé ainsi: je suis, parce que jeffectue» (p. 249-250). Le don existentiel est un don d'être (DumOND, 2007). L'individu qui se donne ne veut pas construire un lien, obtenir une reconnaissance, créer une dette. Simplement, pour être, il a besoin d'être libre de se donner. La relation de travail peut-elle permettre au salarié d'être pleinement ce qu'il est, en donnant librement ce qu'il souhaite donner?

L'originalité de la réflexion présente tient à l'idée que ce sens, recherché par les salariés, peut être exprimé et peut donc être l'objet d'une étude. Dans la continuité des travaux de DUMOND (2007), qui a cherché, dans un récent numéro de Gérer et Comprendre, à identifier des manifestations du don gratuit "dans le regard que les individus portent sur ce quills ont reçu» (p. 65), notre étude s'efforce de comprendre le don existentiel dans le regard que les salariés portent sur ce qui donne sens à leur travail. La question est précisément de savoir si des salariés peuvent s'exprimer sur des expériences de don gratuit et si celles-ci peuvent coexister avec les récits de l'échange.

\section{LA LOGIQUE DE SERVICE DANS UN ÉTABLISSEMENT PUBLIC}

Notre démarche a été entreprise au sein d'un CROUS (Centre Régional des Euvres Universitaires et Scolaires) de province, organisme public français ayant pour vocation de faciliter la vie des étudiants dans les domaines de la restauration, du logement, des bourses, de l'action socioculturelle et de l'ouverture à l'international. Le choix de ce terrain nous paraît pertinent, pour deux raisons.

D'une part, la progression dans l'indice salarial s'y opérant à l'ancienneté et non au mérite, le récit d'une expérience de don peut difficilement être attribué à l'attente d'une rétribution financière qui viendrait, à terme, la récompenser. Il est vraisemblable qu'un salarié allant manifestement au-delà de ce qu'on peut attendre de lui a la sensation de réaliser un don.

D'autre part, dans les organismes de service public, le sentiment d'utilité sociale est souvent recherché dans l'interaction avec l'usager (PIHEL, 2006; GUYON, 2003; FRANCFORT et alii, 1995). En l'occurrence, le don peut être vécu dans la relation aux étudiants.

La méthodologie de recherche choisie est qualitative et de nature exploratoire, en ceci qu'il ne s'agit pas de mesurer l'importance de la logique du don par rapport à celle de la réciprocité marchande, mais d'analyser des récits portant sur des expériences de don, même si ces 
récits ne peuvent rendre compte ni des actes des salariés, ni des raisons réelles pour lesquelles ceux-ci agissent.

L'écoute et la compréhension des expériences qui font sens pour l'individu supposant une connaissance du contexte, nous avons pu nous appuyer sur d'autres actions, auxquelles nous avions participé dans le même secteur, préalablement à notre étude. Au début des années 2000, souhaitant renforcer le contrôle de gestion dans les CROUS, la direction du CNOUS (Centre National des Euvres Universitaires et Scolaires) avait privilégié une démarche ascendante, partant des préoccupations des opérationnels pour enclencher une dynamique de changement progressif. Les premières études de terrain, menées par un cabinet conseil dont nous faisons partie, avaient montré que les problèmes rencontrés par les CROUS relevaient notamment de l'absence d'un projet à moyen terme, qui fût fort, connu et compris par la majorité des personnels, au plan national et régional.

À l'issue de ces études préalables, la direction du CNOUS s'était dite favorable à notre proposition consistant à mettre en place un projet d'établissement dans les CROUS au sein desquels les directeurs manifestaient explicitement leur accord et leur engagement. Nous avions ainsi participé à la construction d'un projet d'établissement au sein de quatre CROUS. À chaque fois, nous avions consulté des données secondaires (rapports d'activité, bilans sociaux, règlement intérieur, tracts syndicaux) et rencontré plusieurs responsables (directeur, agent comptable, responsable des ressources humaines, coordinateur restauration, responsable logements), notamment dans le cadre des groupes de pilotage constitués pour le projet. Nous avions également organisé des entretiens avec le personnel (plus d'une centaine, au total), qui nous ont aidés à conceptualiser notre questionnement sur le don. Nous avons pu aborder ce questionnement à l'occasion de la construction d'un projet d'établissement dans un cinquième CROUS, comptant environ six cents salariés. Des entretiens semi-directifs ont été réalisés auprès d'un échantillon de trente-sept individus. Pour garantir la représentativité de l'échantillon (notamment en ce qui concerne le genre, l'âge et le métier), les répondants ont été sélectionnés selon la méthode des quotas (voir tableau 1).
Le guide d'entretien semi-directif utilisé à cette occasion ne se focalisait pas sur le thème du don. Le premier temps de l'entretien a été organisé autour d'un questionnement, d'ordre général, sur le sens de l'action du CROUS (l'image que les salariés ont du CROUS aujourd'hui et, selon eux, l'axe prioritaire d'amélioration du CROUS à l'avenir). Pour solliciter des témoignages sur d'éventuelles expériences de don, nous avons ensuite demandé aux répondants de décrire des moments forts ou des situations, qui leur procurent - ou leur ont procuré - de la satisfaction, dans le cadre de leur travail. Des questions, telles que "qu'est-ce qui vous donne envie de venir travailler chaque jour?", "Quels sont les projets qui ont suscité votre enthousiasme?" ou encore "Comment décririez-vous, au fond, votre mission?", furent utilisées dans une perspective maïeutique. La logique de réciprocité n'avait pas été exclue de l'entretien, puisque les salariés étaient invités à s'exprimer sur la reconnaissance financière et sociale pour le travail accompli. Les thèmes liés à l'organisation du travail et au management (la définition des rôles de chacun, la diffusion de l'information au sein du CROUS, la relation avec le responsable direct, la présence de l'équipe de direction sur le terrain) ont également été abordés. La dernière partie de l'entretien a pris la forme d'un dialogue, nous permettant de soumettre à la personne interviewée notre propre lecture des expériences qui nous ont été livrées. Cet échange, auquel nous avons convié chacune des personnes interviewées, nous a permis de revenir sur les récits de vie professionnelle, parfois intimes, et de faire valider notre compréhension personnelle par les personnes concernées.

Si tous les points du guide d'entretien ne sont pas directement liés à l'objet de notre recherche, ils enrichissent malgré tout l'analyse, car ils permettent de mieux comprendre le vécu des salariés sur les plans relationnel et organisationnel. Les entretiens, menés sur le lieu de travail, ont duré en moyenne 1 heure 10, et ils ont été enregistrés et retranscrits intégralement. L'enregistrement est une technique à laquelle nous tenions tout particulièrement, en raison de l'importance du vocabulaire utilisé pour cerner avec précision le sens des propos émis (D’IRIBARNE, 1998), en particulier sur les expériences de don.

\begin{tabular}{|l|l|l|l|l|l|}
\hline \multirow{2}{*}{ Métiers } & \multicolumn{2}{|c|}{ Hommes } & \multicolumn{2}{c|}{ Femmes } & \multirow{2}{*}{ TOTAL } \\
\cline { 2 - 5 } & Moins de 45 ans & Plus de 45 ans & Moins de 45 ans & Plus de 45 ans & \\
\hline Restauration & $62(4)$ & $39(3)$ & $85(5)$ & $54(3)$ & $240(15)$ \\
\hline Hébergement & $54(3)$ & $34(2)$ & $74(5)$ & $48(3)$ & $210(13)$ \\
\hline Services administratifs & $38(2)$ & $25(2)$ & $53(3)$ & $34(2)$ & $150(9)$ \\
\hline TOTAL & $154(9)$ & $98(7)$ & $212(13)$ & $136(8)$ & $600(37)$ \\
\hline
\end{tabular}

(Les chiffres mis entre parenthèses concernent l'échantillon interrogé, les autres - la population-mère).

Tableau 1 : Echantillon, établi selon la méthode des quotas 


\section{COMPRENDRE LE DON EXISTENTIEL}

La quasi-totalité des salariés (trente-deux sur trentesept), éprouvent le besoin d'affirmer qu'ils ont apprécié ces moments durant lesquels ils ont agi au-delà du prévisible et sans penser à une éventuelle rétribution sociale ou financière. Quatre observations tirées des entretiens réalisés permettent, non pas de rationaliser le don, qui nous semble échapper à toute objectivation, mais d'affiner l'analyse du don existentiel, dont les dimensions, révélées par GodBOUT (2000), sont l'absence de référence à l'échange et la liberté du don.

\section{Une expérience inattendue}

Les moments forts évoqués par les salariés se rapportent à des expériences à l'occasion desquelles ils ont apporté davantage que ce que la hiérarchie attendait d'eux. Les salariés ont conscience d'avoir éprouvé une satisfaction à agir en toute liberté, indépendamment de contraintes morales ou organisationnelles. Il est notable que certains salariés regrettent que leur supérieur ne conçoive pas qu'ils puissent avoir envie de travailler au-delà de ce qui est normalement attendu et de ce qu'il leur est demandé. Un concierge de cité universitaire, qui accueille les étudiants en souffrance et effectue les petites réparations nécessaires dans les chambres, s'étonne de l'indifférence affichée par son responsable hiérarchique: "Le plus drôle, c'est mon directeur: il n'est au courant de rien, de rien du tout. Je crois que ça ne l'intéresse pas! ». De nombreux salariés partagent le sentiment que leurs initiatives peuvent leur être reprochées ou, tout au moins, susciter une incompréhension. En témoigne un veilleur de nuit de cité universitaire qui, conscient de ne pas être aisément joignable lorsqu'il effectue des rondes dans les trois bâtiments de la cité, a affiché son numéro de portable, pour les cas d'urgence: "Le directeur m'a traité de fou, quand il a vu mon numéro de portable affiché. Il m'a dit: ça va sonner pour un oui ou pour un non. Il est resté bête, quand je lui ai dit que c'était pas un problème, pour moi... "Ces salariés perçoivent leurs initiatives comme des expériences uniques et ils considèrent que le malentendu est en grande partie lié à la difficulté qu'il y a à s'exprimer sur le don. Près d'une dizaine de salariés ont spontanément fait remarquer qu'ils ne s'étaient quasiment jamais confiés sur ce sujet avant le présent entretien. Ce n'est donc pas le désir de témoigner du bien accompli qui conduit certains salariés à vivre des expériences de don, mais bien plutôt une joie intime, spontanée et peu intelligible. Les moments de satisfaction relatés par les salariés n'étaient pas seulement en décalage avec ce que la Direction pouvait attendre: ils ont été vécus par les salariés eux-mêmes comme une aventure, qui leur a permis d'aller au-delà de ce qu'ils apportaient habituellement. Un chef cuisinier d'un restaurant universitaire évoque une initiative ayant consisté à prévoir un thème culinaire différent pour chaque jour de la semaine: "Tous les chefs cuisiniers disent la même chose: "Vraiment, on s'éclate, avec cette nouvelle formule!" Chez moi, je cherche sur Internet des recettes par rapport à nos thèmes. Les étudiants viennent plus nombreux. Et puis, on voit bien quils sont contents. Ça fait vraiment plaisir!» La nouveauté du projet donne au salarié la sensation de pouvoir aller au-delà des tâches normalement effectuées. Le plus souvent, le salarié ne sait pas précisément pourquoi il agit: l'action a sa propre finalité. Une responsable de l'action sociale et culturelle confesse l'irrationalité de son engouement pour certains projets: "Je ne sais pas vraiment pourquoi certains projets miennuyaient, alors que d'autres m'enthousiasmaient. En tout cas, pour ceux qui m'enchantaient, j'ai eu vraiment la sensation de me donner complètement, et ce sont les meilleurs moments de mes dernières années d'activité professionnelle».

\section{Une expérience libre}

Les moments de plaisir évoqués par les salariés se rapportent à un choix libre d'événements, ou d'étudiants, pour lesquels ils ont agi. Un concierge de cité universitaire parle d'un étudiant qu'il souhaite aider et pour lequel il a pris rendez-vous avec une assistante sociale: "Il travaillait tous les soirs chez McDo. Je le voyais rentrer tous les jours vers minuit. Il avait des cernes sous les yeux. Je me suis dit: "toi, mon p'tit père, tu vas pas tenir longtemps comme ça!”... » Une femme de ménage manifeste explicitement son désir d'aider les étudiants qui "ne vont pas bien", qui "ne parlent pas» et dont "les stores sont toujours fermés». Un secrétaire de cité universitaire est particulièrement sensible aux étudiants étrangers, qui ne sont pas toujours bien informés du niveau de confort des logements: "Ils croient trouver des draps, des taies, des verres... C'est vrai dans les cités, mais pas dans les résidences. Dans les résidences, il n'y a rien. C'est pour ça, que je me suis fait une provision de linges et de couverts en plastique, que j'ai ramenés de chez moi : ça sert de dépannage...». Il n'est guère étonnant que les salariés qui ont la possibilité d'avoir des rapports directs avec les étudiants aient été plus prolixes sur la volonté de leur apporter un soutien et sur la satisfaction qu'ils en retirent. La relation de travail semble avoir un sens, parce qu'elle est l'occasion de rendre des services aux étudiants.

\section{Une vision non légaliste du travail}

Les salariés qui ont évoqué des expériences de don éprouvent également le besoin d'exprimer une vision non légaliste, en vertu de laquelle ils ne se satisfont pas du simple respect des règles. Ils manifestent le désir de s'adapter aux situations individuelles, de prendre des initiatives en fonction des besoins et ils témoignent de leur attachement à la rencontre humaine. Ainsi, une secrétaire de cité universitaire regrette que les femmes 
de ménage du CROUS partant en retraite soient remplacées par du personnel de sociétés prestataires de services: "La femme de ménage du CROUS connaît bien les étudiants: elle leur parle, elle signale les problèmes au concierge, etc. Alors que la femme de ménage d'une société externe, ce n'est jamais la même; elle fait son ménage et s'en va [...]». À l'opposé, parmi les cinq salariés qui n'ont pas évoqué d'expérience de don, quatre d'entre eux semblent partager une approche plutôt légaliste du fonctionnement de l'organisation, selon laquelle un travail bien fait signifie le respect des normes prescrites (Alter, 1991). Un agent administratif justifie ainsi sa manière de travailler: "Le CROUS, ça fonctionne surtout grâce aux deniers de l'Etat. Il faut des règles, et les respecter. On ne peut pas toujours rentrer dans les cas particuliers, prendre en compte la situation personnelle des étudiants, sinon, on ne s'en sort plus...».

\section{Une expérience non exclusive}

Les salariés ayant témoigné d'expériences de don ont également, pour la totalité d'entre eux, exprimé leur attachement à la symétrie de l'échange. En particulier, lorsque les salariés analysent l'équilibre de l'échange salarial, ils sont conduits à évoquer leurs attentes et à regretter d'éventuelles asymétries. Par exemple, une salariée cadre, directrice d'un restaurant universitaire, se plaint de la mise en place d'un nouveau logiciel l'obligeant à tenir une comptabilité excessivement précise (à quatre chiffres), qui la prive de toute marge de manouvre: "J'ai passé l'àge, d'être contrôlée dans tout ce que je fais et de me faire tirer les oreilles!». Cette frustration, liée aux difficultés de la relation de travail, ne l'empêche pas de considérer que ses engagements audelà de l'attendu sont une source précieuse de satisfaction: "Je me suis proposée d'être coordinatrice des soirées musicales du vendredi. Je suis passionnée de musique. J'ai des contacts dans le milieu, avec les Asso étudiants. Même si ça me prend du temps (ça déborde souvent sur le weekend), ça me permet vraiment de respirer. Les étudiants sont ravis... ". De même, une salariée non cadre, serveuse en restaurant, qui nous a fait part d'une insatisfaction liée à l'absence de reconnaissance et de rétribution financière, s'est longuement exprimée sur le sens qu'elle prête à son travail: "Plusieurs fois, j'ai essayé de parler de ce que je fais en-dehors des objectifs qui me sont fixés: le chef sénerve et minterrompt. Il pense que je lui demande de l'argent, alors que j'essaie de lui parler de ce qui se passe, de ce que je fais, de ce qui mintéresse, de ce que j'aime, quoi...»

Dès lors que les salariés sont attachés aux deux registres, le recours à la seule logique marchande peut occasionner des malentendus. Ainsi, un chef-cuisinier a mal interprété l'idée d'une prime d'intéressement, dont le montant serait fonction du nombre d'étudiants servis: "Moi, jaime mon boulot. Mon boulot, c'est servir les étudiants. Je ne compte pas les heures. Si on me donne une prime par rapport au nombre de couverts étudiants, ça serait vraiment faire de moi un mercenaire, quelqu'un qui ne pense qu'à l'argent!..."

En ce sens, RICHEBÉ (2002) explique que la mise en place d'une nouvelle classification visant le passage d'une logique de qualification à une logique de compétence a provoqué, chez la majorité des salariés d'une administration, des réactions de mécontentement et l'adoption d'un comportement de retrait, alors que le but était, au contraire, de développer l'apprentissage, la formation, l'envie de coopérer. Cette attitude de retrait s'explique en partie par le fait qu'en donnant, à chacun selon sa contribution effective, la nouvelle classification a été interprétée comme une façon d'attribuer aux salariés une conduite exclusivement intéressée, qui dévalorisait leur travail. Le recours des salariés aux deux registres montre que, s'ils sont attachés à l'équilibre de l'échange, ils sont également conscients du sens que leur apportent leurs expériences de don existentiel.

\section{ACCUEILLIR LE DON EXISTENTIEL}

Il ressort de la présente étude que les dimensions du don existentiel évoquées par GoDBOUT (2000) peuvent être exprimées, certains récits se caractérisant par une absence de référence à un éventuel contredon et par un choix libre d'un tiers-donataire, en présence duquel le don s'opère de façon inattendue. Dans notre étude, ces expériences sont tournées vers les étudiants. Alors que le rapport au supérieur hiérarchique est durable, personnel, impliquant certaines obligations, le rapport à l'étudiant est davantage décrit comme une aventure nouvelle, libre, non réfléchie, ancrée dans l'instant présent: "Il y a, dans le don, de la spontanéité, quelque chose qui se décide spontanément, en-dehors de tout calcul et de toute réflexivité.» (CAILLÉ, 2001, p. 70).

Qu'est-ce que ce don existentiel, qui donne un sens au travail? On concédera qu'il est tentant de voir le don existentiel comme une séquence de la logique du don/contredon (REYNAUD, 1997). Nous sommes bien contraints d'admettre que, dans l'ordre rationnel, "aucun exemple d'action altruiste ne peut réfuter l'idée selon laquelle il en existe une motivation égoïste cachée» (POPPER, 1990, p. 2). On peut toujours déceler un intérêt dans les initiatives bienveillantes des salariés du CROUS. Il y a, ici, un sourire échangé, signe de gratitude, et là, une amertume, qui pourrait prendre le pas sur le don gratuit.

Mais, comme le rappelle Dumond (2007, p. 69), «ce qui fait la gratuité des actes de don, ce n'est pas leur absence d'intérêt, mais le fait que leur finalité se limite à leur réalisation ». À la lumière de la théorie anti-utilitariste, DuMOND (2007) montre que les différentes formes de don gratuit peuvent toujours être réinterprétées par le donateur (ou par un tiers), 
comme du carriérisme (dans une vision transactionnelle) ou comme du service minimal rendu (dans une vision normative): "c'est non seulement le sens, mais l'action induite, qui sinverse" (p. 71).

Aussi, n'écartons-nous pas une autre interprétation des expériences de don: la possibilité même du don parfaitement gratuit. À partir de là, une question d'analyse possible de la relation de travail. Le don gratuit peut occasionner (ou/et renforcer) des asymétries acceptables, pour le bénéficiaire comme pour le donateur, simplement parce qu'il permet d'agir librement. Au final, si la logique du don/contredon est le moteur de l'échange, la logique du don existentiel est ce qui donne profondément sens à la relation de travail.

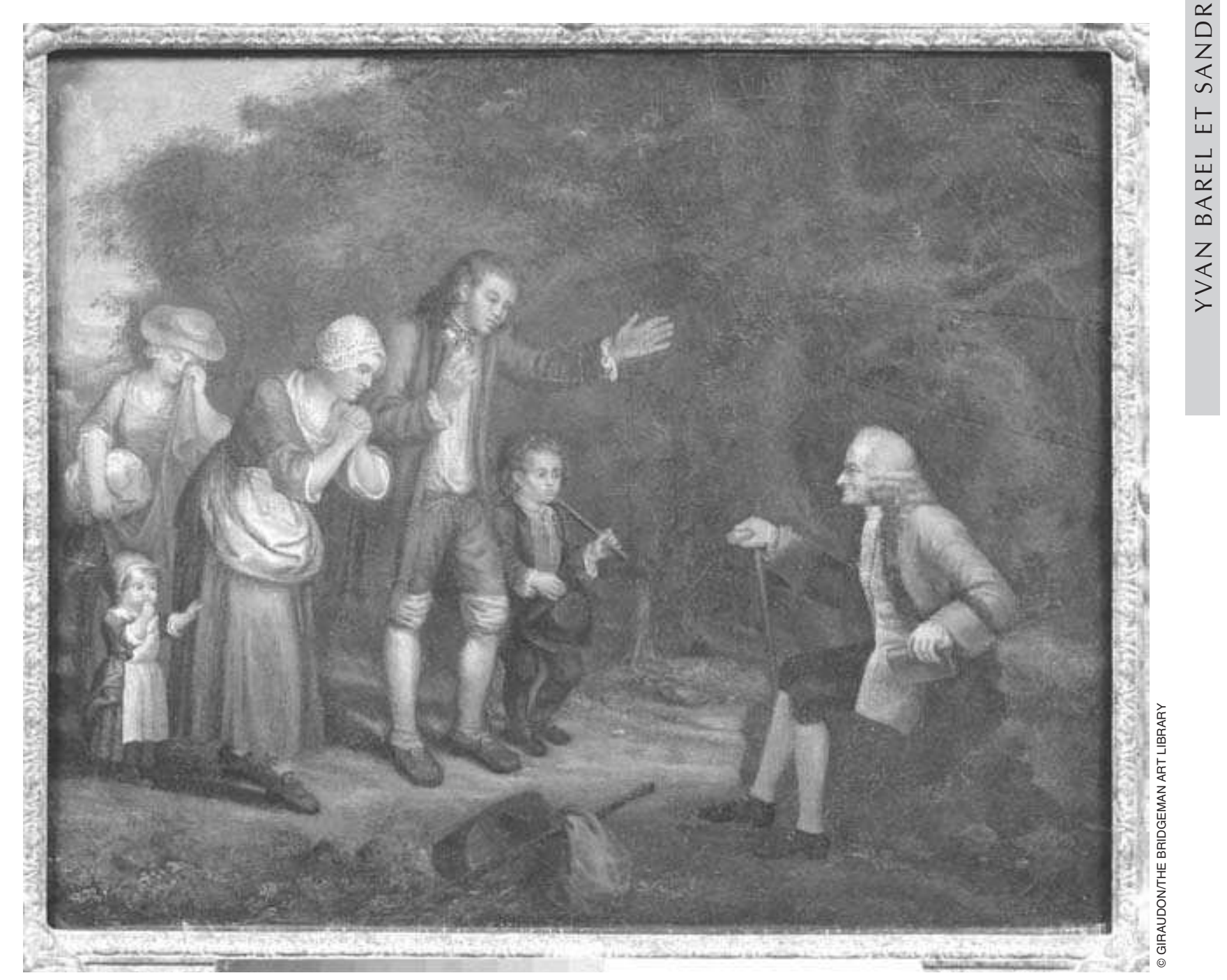

"Le don est porteur de sens parce qu'il témoigne tout à la fois du refus du rapport instrumental à autrui, de la joie de donner et de la portée affective des échanges sociaux». La famille Callas et Voltaire à Ferney, (école française, XVIII siècle, musée Antoine Lécuyer, Saint-Quentin).

demeure: pourquoi est-il si difficile d'admettre et d'accueillir le don gratuit?

La littérature portant sur la logique du don/contredon apporte un élément de réponse: le don serait perçu comme une affirmation de pouvoir (RUCKER, 1991) ou d'individualité (SCHWARTZ, 1967). Aussi, le bénéficiaire du don qui résisterait à la tentation de restituer un don de valeur similaire éprouverait-il un sentiment d'infériorité (SHERRY, 1983) ou de dépendance (MoschetTi, 1979). Afin d'échapper à ce sentiment, il doit réduire (voire supprimer) l'asymétrie de l'échange. C'est sur ce point, que le don gratuit differe de la logique du don/contredon et quili constitue un cadre

\section{CONCLUSION}

La tentation est grande, de considérer que la logique du don gratuit serait hors de portée humaine: c'est comme si on passait brutalement du "trop peu humain» et égoïste au «trop humain» et désintéressé. Bien que cela soit difficile à prouver, il se peut que le don soit, au contraire, ce qui est véritablement humain, et à la portée de tout ce qui est humain.

La tentation est tout aussi grande de faire du don existentiel une réalité - certes possible - mais inaccessible au 
chercheur en sciences de gestion. Il est vrai que la gestion en tant qu' "ensemble des systèmes de transaction» et le don occupent deux espaces bien distincts (DUMOND, 2007). Mais il se peut que le don gratuit soit susceptible, de temps à autre, d'être accessible au langage, et l'analyse des narrations portant sur le don est, dès lors, de nature à enrichir la compréhension du sens des relations dans la sphère professionnelle (REYNAUD, 1997).

S'ouvre alors un nouveau champ de recherche, qui envisage la relation de travail comme une porte d'accès à des expériences de don. D'abord, d'autres terrains de recherche pourraient être exploités. DuMOND (2007) avait choisi d'effectuer son analyse du don gratuit au sein d'une association dont l'objet était d'aider les professionnels à travailler avec des personnes en souffrance psychique. Si les salariés d'un organisme du service public, comme ceux du milieu associatif, semblent être particulièrement sensibles au don, il n'est pas certain que des résultats similaires puissent être obtenus dans d'autres organismes ou entreprises. Ensuite, il nous semble pertinent de nous demander si le manager doit évoquer la possibilité même du don gratuit. Il y a un certain paradoxe, à vouloir témoigner ou rendre compte du don existentiel. S'il était instrumentalisé dans un discours managérial, le don gratuit risquerait d'être dénaturé. Et l'on assisterait, là encore, à une inversion des sens, le don n’apparaissant plus comme ce qui permet d'être, mais comme ce qui donne lieu à reconnaissance. Il est dommageable, de ce point de vue, que la littérature portant sur le leadership se borne à montrer que les dirigeants peuvent "donner du sens» au travail en valorisant le rôle des salariés dans l'organisation et dans la stratégie de l'entreprise. Utiliser le discours managérial dans cette perspective revient à communiquer sur un message que l'École de Palo Alto qualifie de paradoxal, c'est-à-dire sur un message qui, en même temps, nie ce qu'il affirme et affirme ce qu'il nie. Donner du sens est paradoxal, car "le sens ne se donne pas» (MORIN, 2005). Il n'en demeure pas moins qu'il peut être découvert. Il peut même être dévoilé par les dirigeants qui accueillent le don gratuit, en reconnaissant que le sens recherché dans le travail est différent pour chacun des individus (SAinsaulieu, 1977; Dubar, 1991 ; ThÉVENET, 2002). C'est pourquoi la question mérite une attention particulière dans les études futures: le don existentiel, qui relève d'un registre indépendant, mais non opposé à celui de la gestion, peut-il coexister avec la logique de la réciprocité, dans les discours managériaux?

La présente étude ne postule pas que le don existentiel seul existe (ou doive exister) en toute circonstance, mais qu'il n'est pas à exclure, a priori, comme élément d'appréciation du sens prêté au travail. Parce que la logique du don est décrite comme le registre du mouvement vers un tiers, elle peut être l'occasion de mettre en évidence, dans les recherches futures portant sur l'implication et la motivation, l'importance qu'a, pour un grand nombre de salariés, un rapport personnel, libre, non stratégique à la clientèle, à l'usager ou au patient.

\section{BIBLIOGRAPHIE}

AdAMs (J.S.), Inequity in social exchange, in BERKOWITZ (ed.), Advances in experimental social psychology, Academic Press, New York, 1965.

Alter (N.), La Gestion du désordre en entreprise, Paris, L'Harmattan, 1991.

BECKER (H.S.), "Notes on the concept of commitment", American Journal of Sociology, 66, p. 32-40, 1960.

Blau (P.M.), Exchange and Power in Social Life, New York, Wiley, 1964.

BOLTANSKI (L.), L'Amour et la justice comme compétences. Trois essais de sociologie de l'action, Paris, Métaillé, 1990. Bourdieu (P.), Le Sens pratique, Paris, Éditions de Minuit, 1980.

Bourdieu (P.), Raisons pratiques, Paris, Éditions du Seuil, 1994.

Brief (A.P.) \& NoRD (W.R.), Meaning of Occupational Work, Toronto, Lexington Books, 1990.

CAILlÉ (A.), Don intérêt et désintéressement, Bourdieu, Mauss, Platon et quelques autres, Paris, Mauss/La Découverte, 1994.

CAIllé (A.), Anthropologie du don, le tiers paradigme, Paris, Éditions Desclée de Brouwer, 2001.

Caillé (A.) \& Godbout (J.T.), L'Esprit du don, Paris, La Découverte, 1992.

Cordonnier (L.), Coopération et réciprocité, Paris, Presses Universitaires de France, 1997.

Courpasson (D.), L'Action contrainte. Les organisations libérales et la domination, Paris, Presses Universitaires de France, 2000.

D'IrIBARNE (P.), Culture et mondialisation. Gérer pardelà les frontières, Paris, Éditions du Seuil, 1998.

Derrida (J.), "Donner la mort», L'Ethique du don, Jacques Derrida et la pensée du don. (J-M.) RABATE \& (M.) WETZEL, dir. Paris, Métailié-Transition, 1992.

Dubar (C.), La Socialisation, Construction des identités sociales et professionnelles, Paris, Armand Colin Éditeur, 1991.

Dumond (J.-P.), «Oblativité et rationalité instrumentale dans le travail de coopération", Revue Internationale de Psychosociologie, Paris, Eska, n 7, 1997.

Dumond (J.-P.), "Les contradictions entre l'économique et le social à la lumière du don ", Paris, Actes $16^{\mathrm{e}}$ Congrès de l'AGRH, 2005.

DumOnd (J.-P.), «Le don est-il une notion de gestion?", Gérer et comprendre, nº 89, p. 63-72, 2007.

Durkheim (E.), De la division du travail social. Paris, PUF, 1996.

Eisenberger (R.), ARMeli (S.), ReXWINKel (B.), LyNCH (P.D). \& RHOADES (L.), "Reciprocation of perceived organizational support», Journal of applied Psychology, vol. 86, p. 42-51, 2001.

Eustache (D.), «Politique salariale, régulation et échange social», Revue française de sociologie, 42 (2), p. 295-326, 2001. 
Fournier (G.), Bourassa (B.) \& BejI (K.), La Précarité du travail: une réalité aux multiples visages, Québec, Les Presses de l'Université Laval, 2003.

Francfort (I.), Osty (F.), Sainsaulieu (R.) \& Uhalde (M.), Les Mondes sociaux de l'entreprise. Paris, Éditions Desclée de Brouwer, 1995.

Fromm (E.), La Passion de détruire. Anatomie de la destructivité humaine, Paris, Robert Laffont, 1975.

Fryer (D.) \& PAYNe (R.), "Working definitions», Quality of working life, 1 (5), p. 13-15, 1984.

Godbout (J. T.), L'Esprit du don, Montréal, Boréal, 1992. Godbout (J.T.), Le Don, la dette et l'identité, Paris, La découverte, 2000.

GOULDNER (A.W.), «The norm of reciprocity: a preliminary statement", American sociological review, 25, p. 161-178, 1960.

GUYON (C.), Réussir le changement dans le service public, Paris, Éditions Eyrolles, 2003.

Homans (G.), "Social behaviour as exchange", American Journal of Sociology, 63, p. 597-606, 1958.

Maslow (A.H.), Motivation and personality, Harper and Row, New York, 1954.

MAuss (M.), "Essai sur le don. Forme et raison de l'échange dans les sociétés archaïques", L'Année sociologique, tome I, p. 30-186, 1924.

MAUss (M.), The gift: forms and functions of exchange in archaic societies, New York, Norton, 1967.

Mc Clelland (D.C.), AtKinson (J.W.), Clark (R.A.)

\& Lowell (E.L.), The achievement motive, Appleton, Century, New York, 1953.

Morin (E.M.), «Le travail, la santé des personnes et l'efficacité des organisations", 66 ${ }^{\text {th }}$ Canadian Psychological Association Annual Convention, Montréal, 2005.

Moschetti, (G.J.), « The Christmas potlatch: a refinement on the sociological interpretation of gift exchange ", Sociological Focus, 12, p. 1-7, 1979.

PIHEL (L.), La Relation d'emploi durable: approche d'une dynamique d'implication singulière. Analyse du cas France Télécom à partir du paradigme du don/contre don, Thèse de doctorat, Université de Nantes, 2006.

Popper (K.), Le Réalisme et la science, Paris, Éditions Hermann, 1990.

Reynaud (J.-D.), Les Règles du jeu. L'action collective et la régulation sociale, Paris, Armand Colin, Éditeur, $3^{\mathrm{e}}$ édition, 1997.
Richebé (N.), «Les réactions des salariés à la 'logique compétence': vers un renouveau de l'échange salarial", Revue Française de Sociologie, no 43, p. 99-126, 2002.

Rogers (C.R.), Le Développement de la personne, Paris, Éditions Dunod, 1998.

ROUSSEAU (D.) (1990), «New hire perceptions of their own and their employer's obligations: a study of psychological contracts", Journal of Organizational Behaviour, vol. 11, p. 389-400, 1990.

Rucker (M.), Leckliter (L.), Kivel (S.), Dinkel (M.), freitas (T.), Wynes, (M.) \& Prato (H.), "When the thought counts: Friendship, Love, Gift exchanges and gift returns", Advances in consumer Research, vol. 18, p. 528-531, 1991.

Sainsaulieu (R.) L'Identité au travail, Paris, Presses de la Fondation Nationales des Sciences Politiques, 1997.

SCHEIN (E.), "How career anchors hold executives to their career paths", Personnel, vol. 52, p. 11-24, 1975.

SCHeIn (E.), Organizational Psychology, Englewood Cliffs, NJ, Prentice-Hall Inc, 1980.

SCHWARTZ (S.H.) \& SAGIG (L.), «Identifying culturespecifics in the content and structure of Value", Journal of Cross-Cultural Psychology, 26 (1), p. 92-116, 1995.

SChwartZ, (B.), "The social Psychology of the gift», American Journal of Sociology, 73 (1), p. 1-11, 1967.

SHERRY (J.F.), "Gift giving in anthropological perspective", Journal of consumer Research, 10, pp. 157-168, 1983.

TERestChenKo (M.), Un si fragile vernis d'humanité: banalité du mal, banalité du bien, Paris, Mauss/La Découverte, 2005.

TESTARD (A.), «Echange marchand, échange non marchand", Revue française de sociologie, $\mathrm{n}^{\circ}$ 42, p. 719-748, 2001.

THÉVenet (M.), Le Plaisir de travailler, Favoriser l'implication des personnes, Paris, Éditions d'organisation, 2002.

ThéVenet (M.) \& Vachette (J.-L.), Culture et comportements, Paris, Éditions Vuibert, 1992.

TRÉPo (G.) \& Dumond (J.-P.), «La confiance est-elle gérable?", Economies et sociétés, Série de Sciences de gestion, $n^{\circ}$ 8-9, p. 181-195, 1998.

Weil (S.), Oppression et liberté, Paris, Éditions Gallimard, 1955. 